\title{
The uptake and acceptability to patients of cystic fibrosis carrier testing offered in pregnancy by the GP
}

\author{
N E Hartley, D Scotcher, H Harris, P Williamson, A Wallace, D Craufurd, R Harris
}

\begin{abstract}
Objective-To determine the uptake and acceptability of cystic fibrosis (CF) carrier testing when offered to women at the first antenatal booking appointment by their general practitioner.

Setting-Eight general practices in the north west region with a combined patient list size of 42000 .

Design-Offer of carrier screening at first antenatal booking appointment to pregnant women below 14 weeks' gestation; women accepting were alternately allocated to either couple testing (with full disclosure) or stepwise testing.

Subjects-Six hundred and twenty three women were offered CF carrier testing.

Main outcome measures-(1) Acceptance of the offer of CF carrier testing. (2) Acceptability of the test to women following screening, evaluated through (i) postal questionnaire, (ii) semistructured interview.
\end{abstract}

Results-Five hundred and twenty-nine $(84.9 \%)$ women accepted the test; the level of uptake varied across the eight practices (range 11-99\%). In 26/249 (10\%) couple tests no paternal sample was provided. When asked what had influenced their decision to be tested, 59/377 (16\%) women did not refer to $C F$ in their answers and six (2\%) said that they did not feel they could refuse the test. After receiving their results, 368/379 (97\%) women felt that they had made the right decision to be tested, but two carriers and three non-carriers had felt unhappy about testing. Couple testing with full disclosure was associated with lower anxiety levels two weeks after receiving the result for the pregnancy than stepwise testing and $82 / 278(29 \%)$ noncarriers believed that they had no residual risk in relation to $\mathrm{CF}$.

Conclusions-The response from women accepting CF carrier testing was largely positive but a minority of women expressed concern about the test and the way it was offered and a substantial proportion of women were falsely reassured by their "negative" result. Higher levels of acceptance tended to occur in the practices which offered the test there and then rather than giving couples more time to decide about testing. Some women appeared to have accepted the test because of a belief in the importance of test- ing in pregnancy rather than because of the disease in question.

(F Med Genet 1997;34:459-464)

Keywords: cystic fibrosis; screening in pregnancy; general practice

Before the cloning of the cystic fibrosis (CF) gene, ${ }^{1-3} \mathrm{CF}$ carrier testing used linkage analysis and was thus restricted to people with a family history of CF. The subsequent development of PCR technology has made it feasible to offer CF carrier screening to the general population. Extending CF carrier testing beyond families with a family history has raised new issues. How do we know who would want such testing and should it be offered before pregnancy or during pregnancy, in primary care, in the antenatal clinic, or in some other setting? Testing in pregnancy has the advantage of approaching women at a time when they are receptive to the idea of screening. ${ }^{4-6}$ The identification of carrier couples before the end of the first trimester also allows couples the option of an early termination of an affected pregnancy. However, as this test is not specific to pregnancy, offering the test outside pregnancy has the advantage of enabling carrier couples to opt for counselling and consider their reproductive options without the anxiety of an ongoing pregnancy. ${ }^{7-9}$ It would seem preferable to offer testing before pregnancy, ${ }^{10}$ but it is debatable how relevant this is thought to be by people with no previous $\mathrm{CF}$ family history. As well as the timing of the offer of the test, there is also the question of who should offer it: GPs, practice nurses, midwives, antenatal doctors, genetic coworkers, or public health workers.

Consideration should also be given to the method by which the test is offered. Wald ${ }^{11}$ introduced "couple" testing in pregnancy whereby samples are obtained from both partners but only those couples where both partners were found to be carriers were informed of their carrier status. This method has the advantage of concentrating counselling resources on carrier couples and avoids the inevitable time of anxiety when a carrier must wait for the partner's sample to be tested as is the case in stepwise testing. ${ }^{5}$ However, couple testing without full disclosure of the results does not allow for cascade testing in families where only one member of the couple has been found to be a carrier. Resolution of these questions has implications for the large number of 
new genetic screening tests which will probably become available.

This study concentrates on the acceptability of CF carrier testing to participating women and its immediate effect on individual subjects and couples. ${ }^{12} 13$

\section{Methods}

SUBJECTS

Between July 1992 and February 1993, eight north west general practices with a combined list size of 42000 began offering cystic fibrosis carrier screening to pregnant women at the first antenatal booking appointment. GPs were asked to participate for two years. Women were excluded from the study if they booked after 14 weeks' gestation or requested a termination of pregnancy at the booking appointment. As CF gene carrier frequency is highest in the white population ( 1 in 20 in the north west region of England), all but one of the practices selected had a low ethnic minority. However, as it was desirable for GPs to incorporate the offer of the test into their routine antenatal booking appointment, GPs were asked to offer the test to all eligible pregnant women, irrespective of their ethnic background. Appropriate corrections were made to carrier risks using the information provided by GPs about a person's ethnicity.

Women were provided with a leaflet describing cystic fibrosis, its genetic inheritance, and the implications of a positive carrier screening test. Having accepted the offer of the test, women were alternately allocated by the GP either to stepwise or couple testing and were given a second more general leaflet which was specific to the method of testing. Stepwise testing involved the woman providing a mouthwash sample in the surgery. Her partner was only tested if she tested positive. For couple testing the woman provided a sample in the surgery and included in their second leaflet were instructions on how her partner should provide and return a mouthwash sample along with the necessary equipment. Both samples were tested simultaneously.

In order to investigate the possibility that women may not be given enough time to make an informed decision about accepting the offer of the test, practice 7 was asked to offer the test in an alternative way. The intention was to examine whether the uptake was altered by this method. The GP discussed the test with the pregnant woman, who took the information leaflet and test equipment home to explain the test to her partner. The couple then returned both samples to the practice if they wanted the test. In reporting the results of the project, this practice will not be included in any comparison of stepwise and couple testing methods.

Couples with a known family history of $\mathrm{CF}$ who had not previously been CF carrier tested were offered the test, but because of the increased prior risk, GPs were asked to obtain samples from both partners. This avoided unnecessary delay if either sample tested positive. When appropriate one of the genetic associates (DS, NH) visited these couples to clarify their family history and to establish whether a mutation analysis had been carried out in the affected family member.

All samples were sent to the Regional Molecular Genetics Laboratory at St Mary's Hospital, Manchester, where they were tested for the four most common cystic fibrosis mutations in the north west region of England (A Wallace, personal communication). The risk of the person being a carrier and the risk to the pregnancy of $\mathrm{CF}$ were reported in writing to all patients tested and to their respective GPs.

\section{Evaluation}

Two weeks after the test result was posted, women were sent a questionnaire containing 27 questions covering women's feelings about the way the test was offered, their anxiety while waiting for the results, and their knowledge about cystic fibrosis, its inheritance, and their personal carrier risk. Women were also asked to complete a Spielberger State Trait Anxiety Index questionnaire ${ }^{14}$ and during the latter 18 months of the project an additional questionnaire was introduced exploring womens' feelings towards their pregnancy. ${ }^{15}$

Women willing to complete a questionnaire were also asked to agree to be interviewed at home by a genetic associate (DS, NEH). The semistructured interview covered similar areas to the questionnaire but in more detail, as well as more sensitive questions about reproductive intentions that were thought to be inappropriate for inclusion in a postal questionnaire.

\section{Results}

Of the 623 women offered the test, 529 $(84.9 \%)$ women accepted. Two hundred and sixty two were allocated to couple testing and 267 were allocated to stepwise testing. Forty two women declined the test and a further 52 women, having taken information and equipment away, then failed to return samples. In the stepwise group, 10 maternal carriers were found and all their partners were found to be at low risk. In the couple group, seven maternal carriers were found and nine paternal carriers, including the only carrier couple detected. ${ }^{4}$

All except practice 7 were asked to offer the test in the same way. Fig 1 indicates how some GPs modified the original method during the study. The reasons for these modifications ranged from practical issues, such as the time taken to offer the test, to GPs' concerns about population screening and whether informed consent was being given by women before testing and also the direct experience of identifying CF carriers.

LEVEL OF UPTAKE BY PRACTICE

The uptake figures shown in fig 2 relate to the woman's acceptance of cystic fibrosis carrier testing as distinct from accepting a particular method of testing. GPs were not asked to discuss and record the details of why women declined to be carrier tested.

For women accepting the test and allocated to couple testing, the paternal sample was returned by over $90 \%$ of partners in five practices but by only $52 \%$ and $72 \%$ in practices 2 


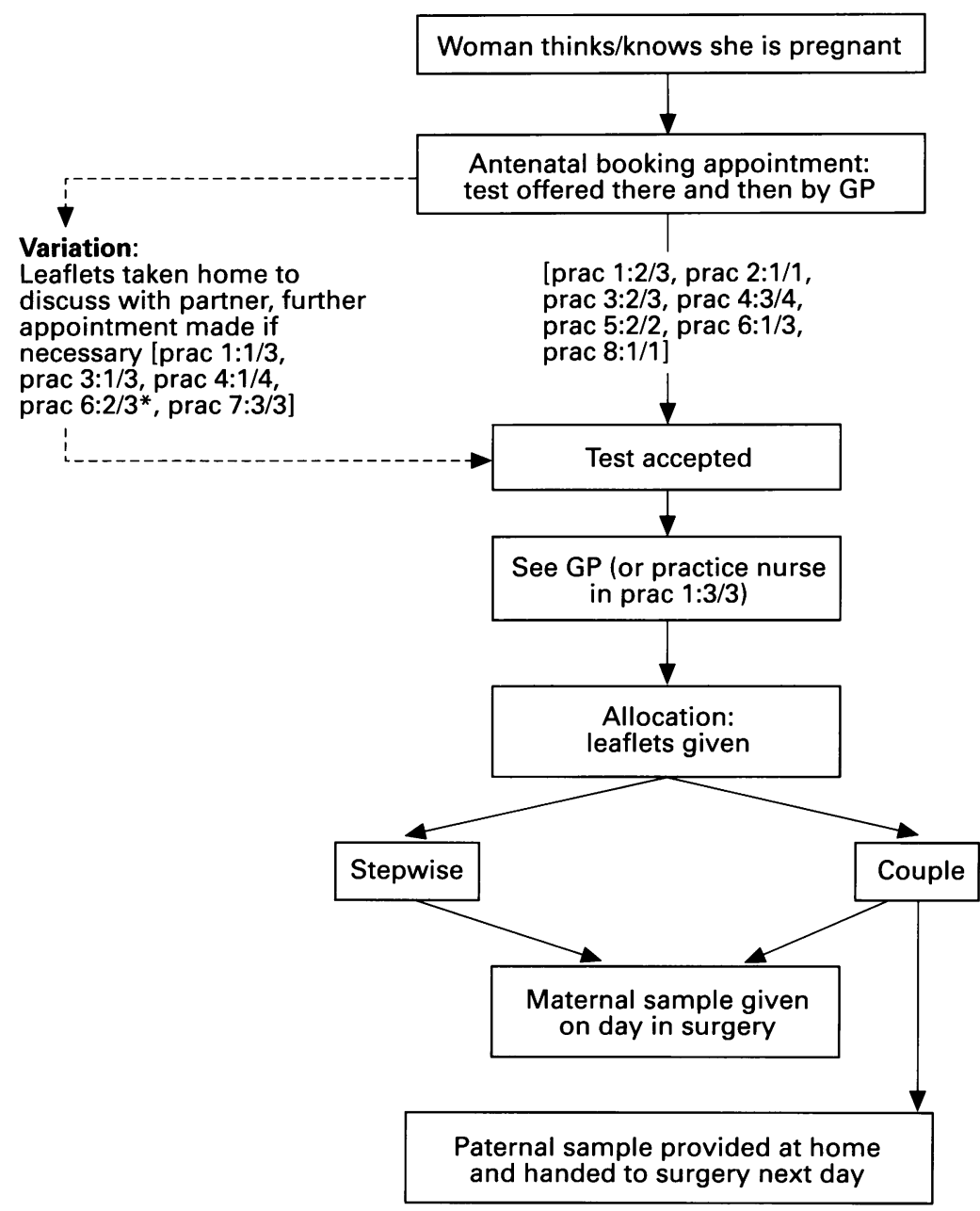

*One of these GPs provided women with tubes and saline and asked them to post their sample (and their partner's where appropriate) directly to the laboratory

Figure 1 Protocol variations in the method of offering cystic fibrosis carrier screening by 20 GPs in eight practices. The practice (prac) number is followed by the proportion of GPs in that practice who offered the test in a particular way. One GP not wishing to participate has been excluded from this figure.

and 3, respectively. In 12 of the 26 cases where a paternal sample was not returned the women either declined or did not respond to follow up so no information about the partner's sample was available. In the remaining couples the maternal interview and questionnaire data showed that four of the 14 partners "didn't get round to it" and in $2 / 14$ the sample was not received by the laboratory. In eight cases the partner either declined or was reluctant to give a sample.

CHARACTERISTICS OF WOMEN TAKING THE TEST The median age at the time of testing was 27 years (range 15-42) with $96 \%$ giving their ethnicity as white. Comparable data were not collected from women declining the test. The median gestation at the time of testing was seven completed weeks.

\section{RESPONSE BIAS}

Questionnaires were not sent to seven women who had miscarried, one woman who had had a termination of pregnancy since providing the sample, one woman who had a family history of $\mathrm{CF}$, one woman in the stepwise group whose sample failed to give a result and who did not provide a repeat sample, and one woman found to be in a carrier couple. A total of 382 completed questionnaires were returned. The difference between questionnaire completion rates in the stepwise $(79 \%)$ and couple $(85 \%)$ groups was of borderline statistical significance $(p=0.06)$. A total of 305 women also agreed to be interviewed of whom 13 failed to complete the questionnaire.

There were 123 women from whom no questionnaire or interview data were obtained; for 35 it was not appropriate either because the woman had subsequently miscarried, had a termination of pregnancy, or was unwell, a further 60 declined follow up, and for 28 the reason was unknown. All but one of the 14 maternal carriers whom it was appropriate to approach provided either questionnaire or interview data or both (93\%) compared to $82 \%$ of maternal non-carriers.

REASONS FOR UPTAKE OF TEST

Women were asked to indicate which, if any, of the factors suggested had influenced their decision to take the test (table 1). All six women who only ticked the option "I did not feel I could refuse the test" were asked to do the 
Practice 1

Practice 2

Practice 3

Practice 4

Practice 5

Practice 6

Practice 7*

Practice 8

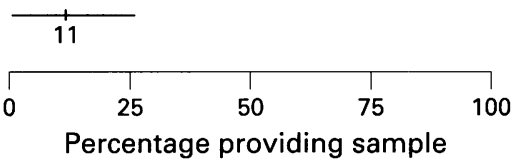

Figure 2 Uptake of cystic fibrosis carrier testing by practice. Vertical line indicates estimate of percentage accepting, horizontal line indicates $95 \%$ confidence interval for estimate. ${ }^{\star}$ In this practice, percentage accepting is where both maternal and paternal samples were provided.

Table 1 Factors influencing the decision to have the test

Factors listed in questionnaire

(a) You feel that all tests offered in pregnancy are importan

(b) You wanted to know if you were a carrier of the cystic fibrosis gene

(c) You were sure that you didn't want to have a child with cystic fibrosis

(d) You didn't feel that you could refuse the test

(e) Your partner was keen for you to have the test

(f) It's difficult to remember now

(g) None of the reasons given

Categorised response

Answer included a reference to CF (b or c)

Answer did not include a reference to $\mathrm{CF}$ but indicated

that woman felt all tests in pregnancy were important

(a but not b or c)

Answered only that they did not feel they could refuse

the test ( $\mathrm{d}$ only)

Answered only that none of the reasons given applied to them $(\mathrm{g})$

Total

No of women

$304(81 \%)$

$59(16 \%)$

$6(2 \%)$

$8(2 \%)$

$377(100 \%)$
Table 2 Decision making

\begin{tabular}{lll}
\hline $\begin{array}{l}\text { What would you do if you and your partner both were found } \\
\text { to be carriers? }\end{array}$ & $\begin{array}{l}\text { Maternal } \\
\text { non-carrier (\%) }\end{array}$ & $\begin{array}{l}\text { Maternal carrier } \\
\text { (\%) }\end{array}$ \\
\hline Not thought about situation at all & $34(12)$ & $4(25)$ \\
Did not know & $6(2)$ & $0(0)$ \\
Wait for results before making decision & $113(40)$ & $4(25)$ \\
Would consider further testing & $84(30)$ & $6(38)$ \\
Would not consider termination & $46(16)$ & $2(13)$ \\
Total & $283(100)$ & $16(100)$ \\
\hline
\end{tabular}

Table 3 Anxiety while awaiting test result for pregnancy (measured two weeks after result known): comparison between couple and stepwise groups

\begin{tabular}{lll}
\hline $\begin{array}{l}\text { We would like to know how you felt while waiting for the } \\
\text { results of the test, compared to how you usually feel, } \\
\text { were you ..... }\end{array}$ & $\begin{array}{l}\text { Stepwise } \\
\text { non-carriers (\%) }\end{array}$ & $\begin{array}{l}\text { Couple carriers and } \\
\text { non-carriers (\%) }\end{array}$ \\
\hline Much more worried than usual & $3(2)$ & $6(3)$ \\
A bit more worried than usual & $54(31)$ & $65(35)$ \\
No more worried than usual & $109(63)$ & $115(61)$ \\
Less worried than usual & $6(4)$ & $2(1)$ \\
Total & $172(100)$ & $188(100)$ \\
\hline
\end{tabular}

test there and then in the GP's surgery and there is no indication that they had been given the option of more time to decide about having the test. At interview, three of these women, including one maternal carrier found in the stepwise group, reported having felt unsure about doing the test, while the remaining three had no regrets about being tested.

DECISION MAKING

From the questionnaire completed two weeks after receiving their result, $368 / 379(97 \%)$ women replied that they did feel they had made the right decision to have the test, six did not know, and five said they felt they had not made the right decision by having the test. In this last group, two of the women were found to be carriers and felt generally unhappy about the whole testing procedure, two found to be noncarriers had expressed uncertainty and concern about the test and neither couple would have opted for further testing in pregnancy, while at interview the remaining one woman appeared to be happy with all aspects of the test. Of the six women who had expressed doubt about their decision to be tested, three felt that they had needed more time to discuss the test and to make up their mind about being tested, one woman had agreed to be tested mostly on the grounds of helping research and had reservations about tests in pregnancy, and two had reported feeling more anxious than normal while waiting for the results.

The responses at interview given by carriers and non-carriers to the question "What would you have done if you and your partner were both found to be carriers?" are shown in table 2.

\section{ANXIETY}

Waiting for the result

The median time between the maternal sample being provided and the result for the pregnancy being communicated to the couple was nine days for stepwise non-carriers and 11 days for both stepwise carriers and all couple tests. If a maternal carrier was found in the stepwise group, the communication was by telephone whenever possible, in order to obtain a paternal sample quickly. The 10 women found to be carriers in the stepwise group then had to wait for a median of two days (range 1-8 days) for the respective paternal sample result.

The level of anxiety experienced while waiting for the test result (table 3) did not differ significantly for women allocated to stepwise testing subsequently found to be non-carriers compared with women allocated to couple testing (chi-square trend test statistic $=2.0$, $p>0.1$ ). Maternal carriers found in the stepwise group are excluded from this analysis because of ambiguity about whether they recalled their anxiety while waiting for their own result or for that of their partners. Nine other cases are excluded from this analysis since no answer was given.

Two weeks after receiving result

Table 4 shows results for state anxiety level approximately two weeks after receiving a final 
Table 4 Spielberger state anxiety scores and attitudes to pregnancy, measured two weeks after result known

\begin{tabular}{|c|c|c|c|}
\hline \multirow[b]{2}{*}{ Measure } & \multicolumn{2}{|c|}{ Method of screening } & \multirow{2}{*}{$\begin{array}{l}\text { Difference between methods } \\
\text { ( } 95 \% \text { confidence interval) }\end{array}$} \\
\hline & Stepwise & Couple & \\
\hline \multicolumn{4}{|l|}{ "State" anxiety } \\
\hline Mean (SD) & $36.9(11.3)$ & $33.3(8.7)$ & $3.6(1.3-5.9)$ \\
\hline No of women (\% responding) & $144(60)$ & $168(73)$ & \\
\hline \multicolumn{4}{|l|}{ "How you feel about being pregnant" } \\
\hline \multicolumn{4}{|l|}{ Positive attitude } \\
\hline Mean (SD) & $16.0(4.2)$ & $15.9(3.8)$ & $0.1(-1.0-1.3)$ \\
\hline \multicolumn{4}{|l|}{ Negative attitude } \\
\hline Mean (SD) & $7.6(6.4)$ & $6.7(5.1)$ & $0.9(-0.7-2.5)$ \\
\hline No of women (\% responding) & $93(39)$ & $109(48)$ & \\
\hline
\end{tabular}

Table 5 Perception of test results by non-carriers: comparison between couple and stepwise groups

\begin{tabular}{|c|c|c|c|}
\hline \multirow{2}{*}{$\begin{array}{l}\text { Method of } \\
\text { allocation }\end{array}$} & \multicolumn{2}{|c|}{$\begin{array}{l}\text { What were the results of your } \\
\text { screening test? }\end{array}$} & \multirow[b]{2}{*}{ Total (\%) } \\
\hline & Low risk (\%) & No risk (\%) & \\
\hline Couple & 99 (69) & $45(31)$ & $144(100)$ \\
\hline Stepwise & $97(72)$ & $37(28)$ & $134(100)$ \\
\hline Total & $196(71)$ & $82(29)$ & $278(100)$ \\
\hline
\end{tabular}

test result. Couple testing with disclosure appears to be associated with lower state anxiety levels than stepwise testing ( $t$ statistic $=3.1$, $\mathrm{p}=0.002$ ). This difference in state anxiety level may reflect a chance difference in the women in the two groups before they were allocated. This assessment was not made at entry to the study.

\section{Feelings about being pregnant}

Results from the completed questionnaire "How you feel about being pregnant" are shown in table 4 . No statistically significant difference was found between either positive or negative attitudes towards being pregnant by women in the stepwise and couple testing groups (positive attitude: $t$ statistic $=-0.2,200$ df, $\mathrm{p}>0.1$; negative attitude: $t$ statistic $=-1.1$, $200 \mathrm{df}, \mathrm{p}>0.1$ ).

\section{PERCEPTION OF RISK}

Overall, $82(29 \%)$ of the 278 non-carrier women interviewed believed that they had no residual risk in relation to $\mathrm{CF}$ (table 5). There is no evidence to suggest that women in either the stepwise or couple group are more falsely reassured (chi-square statistic $=0.441, p>0.1$ ). Of 12 maternal carriers responding, all correctly recalled their carrier status.

\section{FAILED SAMPLES}

Sixteen (2\%) of 776 samples tested failed to give a result. Of these, 12 were paternal samples produced from couple testing. Feedback from laboratory staff indicated that the majority of these had failed to produce an adequate amount of DNA, possibly owing to non-adherence to instructions provided for production of a sample.

\section{Discussion}

On the whole the overwhelming response from women who accepted CF carrier screening by their GP in early pregnancy has been positive, with the majority of women stating that they were given enough information, enough time with their GP, and enough time to decide about being tested. Using the GP booking appointment to offer CF carrier screening, however, is not without its difficulties. A handful of women commented that they had felt rushed when making their decision about being carrier tested. The study as a whole has provided valuable information on genetic screening in the general practice setting.

The level of uptake of the offer of cystic fibrosis carrier testing by the GP varied between practices in this study. This points to a number of areas where further studies might be of interest. Although the way in which the test was offered varied between practices, the study was not designed to be a randomised trial of methods of offering testing and the method is consequently confounded by other practice variables. However, it was noted that the higher rates of acceptance were in four practices in which the test was offered there and then (practices 2, 3, 4, and 5), compared to the lower rates found in practices 1,6 , and 7 where most women/couples were asked to go away and think about the offer before doing the test. Similar results were found in GP surgeries where $453 / 649$ (70\%) non-pregnant people who were offered a test there and then using active recruitment accepted the offer to be tested, while those invited to attend for testing using a passive recruitment method had a lower acceptance rate of $59 / 502(12 \%){ }^{7}$ Variation in the return of paternal samples was also noted among practices in this study, with a lower return rate for two practices (2 and 3 ). There were no obvious reasons for this variation in this study.

It is not possible in this study to establish how important a part the method of offering testing was in a woman's decision to accept. It appears that for $16 \%$, agreeing to be tested was based on their belief that "all tests in pregnancy are important" rather than because they were relating the test specifically to cystic fibrosis. It may be that these women made a decision before seeing their GP that they would accept any and all tests offered and CF carrier testing was not distinguished as being any different. It could be argued that those women accepting the offer of cystic fibrosis carrier testing are part of a susceptible population who are compliant and amenable to testing in general. At interview, over $80 \%$ of women in each practice said that they would have other screening tests offered to them in pregnancy. However, across practices, the numbers responding with the questionnaire did not reflect the pattern in uptake areas.

Two weeks after receiving their result, $97 \%$ of women felt they had made the right decision to have the test. However, when asked at interview what they would have done if they had been found to be in a carrier couple, $16 \%$ of women said they would not have contemplated prenatal diagnostic tests or a termination of an affected pregnancy. This causes concern as to whether these women had appreciated that a positive prenatal diagnosis and offer of termination of pregnancy might be a possible outcome. The finding that $113(40 \%)$ noncarriers and four $(25 \%)$ carriers said that they 
would have waited for the result that they were a carrier couple before making any decisions implies that these women may have decided to postpone decision making until the need arose rather than indicating a lack of informed consent before testing. A similar result was found by Sjogen and Uddenberg ${ }^{16}$ in a study of decision making during the prenatal diagnosis procedure. Although having received their results most women reported the testing procedure to have been acceptable, in retrospect 46 of the 305 women interviewed (15\%) would have preferred the option of a more leisurely consideration of the implications of carrier testing in the absence of an ongoing pregnancy.

In this study current (state) levels of anxiety in women two weeks after receiving the final results for the pregnancy were found to be higher following stepwise testing than couple testing. Anxiety scores were obtained for 144 $(60 \%)$ compared to $168(73 \%)$ of women in the stepwise and couple testing groups, respectively. The validity of the comparison of anxiety scores rests on the assumption that whether the woman responds or not does not depend on her anxiety state. In a study of pregnant women undergoing prenatal screening for fetal abnormality, ${ }^{15}$ no association was found between the initial state measure of anxiety and whether all subsequent STAIs were returned for women who completed an initial questionnaire. This observation lends limited support to the hypothesis that more anxious women, as measured using the current (state) anxiety score, were no more or no less likely to have returned the questionnaire in the current study.

The alternative allocation of women by GPs to stepwise and couple testing was quasirandom. It is possible that a GP may have allocated a woman preferentially, for example, to stepwise testing if she was in difficult social circumstances. This situation may be associated with a higher general level of anxiety. However, after exclusion of patients who were offered the test by GPs with unequal allocation rates, couple testing with disclosure remained associated with a lower state anxiety level $(t$ statistic $=-2.51,226 \mathrm{df}, \mathrm{p}=0.01)$. This difference did not therefore appear to be as a result of biased allocation, although the possibility that the groups were unequal at entry could not be excluded.

This result, although similar in magnitude, contrasts with the findings from a previous randomised trial ${ }^{5}$ where pregnant women receiving a negative result following stepwise screening were less anxious than those who had couple screening. There was a difference between the studies, however, in the disclosure of results for the couple testing method. In the previous study, individual carrier risks were not given, meaning that negative results were given to everyone except carrier couples. In the current study there was full disclosure of all test results to people as well as a risk to the pregnancy. For women whose partners have not been tested, uncertainty about their partner's carrier status could be reflected in raised current (state) anxiety. Conversely, couples where both partners are tested and there is full disclosure of both results might be expected to exhibit lower levels of anxiety. However, although statistically significant, the clinical importance of a three point difference measured on the anxiety index is open to debate.

The majority of women who provided comments four weeks after testing realised that there was a residual risk associated with a "negative" test result, but $29 \%$ understood there to be "no risk". This false feeling of reassurance was not associated with a particular method of testing, in contrast to the study by Miedzybrodzka et at where "couple" testing did seem to be associated with more false reassurance.

It is uncertain whether population cystic fibrosis carrier screening will ever be advocated or implemented on a national scale, but the results from this study will contribute to the debate. There is no easy way to apportion the finite resources available for both screening and treatment of this disease. At some time in the future, attitudes to population screening are liable to be modified further with the prospect of gene therapy or other effective treatments.

We thank the general practitioners, the attached staff, and the many women and their partners who participated in this project. The project was funded by the Wolfson Foundation.

1 Kerem B, Rommens JM, Buchan JA, et al. Identification of the cystic fibrosis gene: genetic analysis. Science 1989;245:1073-80

2 Riorden JR, Rommens JM, Kerem B, et al. Identification of the cystic fibrosis gene: cloning and characterisation of complementary DNA. Science 1989;245:1066-73.

3 Rommens JM, Iannuzzi MC, Kerem B, et al. Identification of the cystic fibrosis gene: chromosome walking and jumping Science 1989;245:1059-65.

4 Harris H, Scotcher D, Hartley NE, Wallace A, Craufurd D, Harris R. Cystic fibrosis carrier testing in early pregnancy by general practitioners. $B M \mathcal{F}$ 1993;306:1580-3.

5 Miedzybrodzka ZM, Hall MH, Mollison J, et al. Antenata screening for carriers of cystic fibrosis: randomized trial of stepwise vs couple screening. BMF 1995;310:353-7.

6 Mennie M, Compton M, Gillfillan A, et al. Prenatal screening for cystic fibrosis: psychological effects on carriers and their partners. $\mathcal{F}$ Med Genet 1993;30:543-8.

7 Bekker H, Modell M, Dennis SG, et al. Uptake of cystic fibrosis carrier testing in primary care: supply push or fibrosis carrier testing in primary car
demand pull ? BMF 1993;306:1584-6.

8 Watson EK, Mayall E, Chapple J, et al. Screening for carriers of cystic fibrosis through primary care services. $B M^{\prime}$ 1991;303:504-7.

9 Payne Y, Williams M, Cheadle J, et al. Carrier screening fo cystic fibrosis in general practice: experience in South Wales. Report to Cystic Fibrosis Research Trust 1994

10 Raeburn JA. Screening for carriers of cystic fibrosis screening before pregnancy is needed. BMF 1994;309: 1428-9.

11 Wald NJ. Couple screening for cystic fibrosis. Lancet 1991;338: 1318-19.

12 Harris H, Scotcher D, Hartley NE, Wallace A, Craufurd D, Harris R. Pilot study of the acceptability of cystic fibrosis carrier testing during routine antenatal consultation in general practice. $B r \mathcal{F}$ Gen Pract 1996;46:225-7.

13 Axworthy D, Brock JH, Bobrow M, Marteau TM. Psychological impact of population-based carrier testing for cystic fibrosis: three year follow-up. Lancet (in press).

14 Spielberger CD, Gorsuch RL, Lusherne RE. Manual for the state-trait anxiety inventory (self evaluation questionnaire). Palo Alto, California: Consulting Psychologists Press, 1970.

15 Marteau TM, Cook R, Kidd J, et al. The psychologica effects of false-positive results in prenatal screening for fetal effects of false-positive results in prenatal screening for feta abnormatic

16 Sjogen B, Uddenberg N. Decision making during the prenatal diagnostic procedure. A questionnaire and interview study of 211 women participating in prenatal diagnosis. Prenat Diagn 1988;8:263-73. 